Palavras chave:

Equações de volume Equações hipsométricas

Espécies nativas

Relações altura-diâmetro

Histórico:

Recebido 31/01/2012

Aceito $31 / 08 / 2014$

Keywords:

Volume equations

Volumetric equations

Native species

Height-diameter relationships

Correspondência: danilo.scorzoni@gmail.com
Danilo Scorzoni Ré', Vera Lex Engel', Liz Miyo Sousa Ota', Luiz Alberto Blanco Jorge'

\section{EQUAÇÕES ALOMÉTRICAS EM PLANTIOS MISTOS VISANDO À RESTAURAÇÃO DA FLORESTA ESTACIONAL SEMIDECIDUAL}

RESUMO: Visando a suprir a lacuna do conhecimento existente sobre a estimação precisa do potencial de produção madeireira de sistemas de restauração florestal, com o presente trabalho, objetivou-se modelar as relações alométricas (hipsométricas e volumétricas) de nove espécies comuns a modelos alternativos de restauração da floresta estacional semidecidual. Os modelos se ajustaram bem aos dados coletados e a análise de resíduos indicou distribuição satisfatória, tanto para os modelos hipsométricos como volumétricos. O modelo hipsométricos de Stoeffels e o modelo volumétrico de Schumacher-Hall foram os mais flexíveis, ajustando melhor para várias espécies. Essas equações obtidas serão usadas em futuros estudos de manejo e conservação de áreas em restauração.

\section{TREE ALLOMETRIC EQUATIONS IN MIXED FOREST PLANTATIONS FOR THE RESTORATION OF SEASONAL SEMIDECIDUOUS FOREST}

ABSTRACT: Aiming to overcome the lack of knowledge about precise estimation of wood production potential of forest restoration systems, the objective of this study is modeling allometric relationships of nine tree species in alternative models of seasonal semideciduous forest restoration. The models fitted well to the data with satisfactory distribution of the residuals. The height-diameter relationship was best modeled by the Stoeffles model and the volume equations had the best fit with the Schumacher-Hall model, for many species. The equations obtained will be used in future studies of management and conservation of these plantations. 


\section{INTRODUÇÃO}

A Floresta Estacional Semidecidual é um dos principais ecossistemas associados ao bioma Mata Atlântica. Historicamente, esse bioma tem sido ameaçado pelo homem desde os tempo coloniais (DEAN, 2007), no entanto, nas últimas décadas, alguns esforços de restauração têm sido realizados para tentar reverter sua destruição (MELO; DURIGAN, 2007) utilizando-se diferentes abordagens . A principal barreira a esses esforços tem sido o alto custo de implantação e manutenção inicial dos projetos de restauração, principalmente quando a ênfase está no alcance de metas apenas ecológicas ou serviços ambientais indiretos (ENGEL, 2003; LAMB et al., 2005). Recentemente, alguns estudos têm sido realizados para propor novos modelos de restauração florestal que permitam o manejo da floresta no futuro e, consequentemente, a geração de bem diretos como madeira, para que o produtor rural possa, pelo menos, pagar pelos custos de implantação do projeto (LAMB et al., 2005; RÉ, 20I I). Para avaliar essa efetividade desses modelos, é preciso rever o potencial de manejo dessas áreas, por meio da quantificação dos recursos madeireiros.

As relações entre altura e diâmetro são muito úteis na descrição de plantações florestais e seu desenvolvimento ao longo do tempo, na estimação de alturas médias e índices de sítio (CURTIS, 1967). Já, as equações volumétricas constituem a principal ferramenta para estimar o volume individual de madeira da árvore em pé, informação fundamental nos planos de manejo em florestas nativas e de suprimento de madeira em florestas plantadas. Essa informação permite, também, o estudo de crescimento e produção de povoamentos florestais (SCOLFORO, 2005).

As equações alométricas, em geral, foram bem desenvolvidas para espécies exóticas de rápido crescimento no Brasil, principalmente do gênero Pinus, Eucalyptus e Acacia (BARROS et al., 2002; FINGER et al., 2000; TONINI et al., 2005; TONINI; SCHWENGBER, 2006; ZANON et al., 1996). As espécies nativas são, muitas vezes, deixadas de lado pela inexistente cultura florestal dos produtores no Brasil e pela inexistência de informações relativas à sua ecologia, silvicultura e biometria (ENGEL, 2003; TONINI et al., 2005). Os poucos estudos com espécies nativas se restringem, em sua maioria, ao desenvolvimento de equações alométricas para florestas do bioma amazônico (COLPINI et al., 2009; SILVA; CARVALHO, 1984; SILVA et al., 1984), bioma Cerrado (IMAÑA-ENCINAS et al.,
2009) e plantios homogêneos (TONINI et al., 2005). Iniciativas de estudos em ecossistemas de mata atlântica são poucos (BATISTA et al., 2004; CHICHORRO et al., 2003; SCOLFORO et al., 1994) e em áreas de restauração da mata atlântica praticamente inexistem.

Objetivou-se, com este trabalho, modelar as relações hipsométricas e volumétricas de nove espécies nativas implantadas em diferentes sistemas propostos, aos 13 anos de idade, para a restauração da floresta estacional semidecidual na região centro-sul do estado de São Paulo.

\section{MATERIAL E MÉTODOS}

\section{Área de estudo e delineamento experimental}

A área experimental situa-se na região centrosul do Estado de São Paulo, município de Botucatu, nas Fazendas Experimentais Edgárdia e Lageado, pertencentes à Faculdade de Ciências Agronômicas, campus da Universidade Estadual Paulista Júlio de Mesquita Filho (UNESP). As coordenadas geográficas aproximadas são $22^{\circ} 50^{\prime} \mathrm{S}$ e $48^{\circ} 24^{\prime} \mathrm{W}$. A zona de vegetação é classificada como floresta tropical estacional semidecidual (INSTITUTO BRASILEIRO DE GEOGRAFIA E ESTATÍSTICA - IBGE, I993), com cerca de $1300 \mathrm{~mm}$ de precipitação média anual, distribuída, principalmente, entre os meses de outubro a março. A temperatura média anual é de $19,4^{\circ} \mathrm{C}$, variando de $21,9^{\circ} \mathrm{C}$ em janeiro a $16,3^{\circ} \mathrm{C}$ em julho. A área é de topografia acidentada, com altitude variando de $464 \mathrm{~m}$ a 775 m. Em decorrência da topografia e embasamento geológico, existe uma variação de tipos de solo, desde mais férteis de origem basáltica (Nitossolo Vermelho - Sítio Lageado), até manchas de solo extremamente pobres e ácidos (Latossolo Vermelho-Amarelo álico Sítio Edgárdia), representando bem a variação do Estado de São Paulo.

Entre 1997 e 1998, implantou-se um experimento de restauração florestal testando quatro modelos de plantio. Maiores informações sobre a caracterização de cada um dos tratamentos pode ser obtida em Ré (20II).

\section{Coleta e análise de dados}

As espécies mais abundantes nos tratamentos, constatadas em censo florestal realizado em 2008 e 2009, foram selecionadas para se realizar a cubagem rigorosa dos indivíduos (Tabela 1), utilizando-se um 
pentaprisma de Wheeler com hipsômetro Suunto acoplado, considerando-se seções de I metro de comprimento. Os volumes das seções foram calculados pelo método de Smalian até o diâmetro mínimo de $5,0 \mathrm{~cm}$; o volume desse ponto até o topo da árvore foi calculado, utilizando-se a fórmula do cone. Com o hipsômetro Suunto, foram tomadas as alturas totais para o cálculo do volume da última seção (cônica) e para ajuste das equações hipsométricas.

TABELA 1 Médias e desvios padrão (parêntesis) de variáveis das árvores amostradas na realização da cubagem rigorosa.

TABLE 1 Means and standard deviations (parenthesis) of the variables sampled for volume determination.

\begin{tabular}{lcccc}
\hline ID Espécie & $n$ & $\bar{d}(\mathrm{~cm})$ & $\bar{h}(\mathrm{~m})$ & $\bar{v}\left(\mathrm{~m}^{3}\right)$ \\
\hline \multirow{2}{*}{ A Anadenanthera macrocarpa } & 110 & 17,5 & 12,7 & 0,184133 \\
& & $(8,27)$ & $(4,54)$ & $(0,191724)$ \\
B Enterolobium contorstisiliquum & 256 & 17,4 & 10,2 & 0,137349 \\
& & $(10,2)$ & $(3,35)$ & $(0,132823)$ \\
C Peltophorum dubium & 114 & 15,4 & 12,3 & 0,114580 \\
& & $(12,3)$ & $(3,44)$ & $(0,095647)$ \\
D Pterogyne nitens & 146 & 13,7 & 11,0 & 0,081720 \\
& & $(11,0)$ & $(2,67)$ & $(0,073604)$ \\
E Schizolobium parahyba & 96 & 21,6 & 15,6 & 0,301845 \\
& & $(15,6)$ & $(4,60)$ & $(0,215061)$ \\
F Zeyheria tuberculosa & 26 & 10,3 & 8,7 & 0,042762 \\
& & $(8,7)$ & $(2,45)$ & $(0,046831)$ \\
G Cedrela fissilis & & 10,9 & 8,0 & 0,043909 \\
& 72 & $(8,0)$ & $(2,23)$ & $(0,039964)$ \\
H Copaifera langsdorffii & 26 & 8,4 & 7,5 & 0,022271 \\
& & $(7,5)$ & $(2,06)$ & $(0,023441)$ \\
& & 9,0 & 8,7 & 0,031625 \\
I Hymenaea courbaril & 161 & $(8,7)$ & $(2,23)$ & $(0,036514)$ \\
\hline
\end{tabular}

$\mathrm{ID}=$ identificação da espécie; $n=$ número de indivíduos amostrados; $\bar{d}$ = média dos diâmetros a $1,30 \mathrm{~m}$ de altura; $\bar{h}=$ média das alturas totais; $\bar{v}=$ média dos volumes individuais; os valores entre parêntesis são os desvios padrão de cada variável.

$\mathrm{ID}=$ specie identification; $\mathrm{n}=$ number of trees sampled; $\bar{d}=$ mean diameter at breast height; $\bar{h}=$ mean total height; $\bar{v}=$ mean individual volume.

Os modelos utilizados no ajuste das relações hipsométricas estão apresentados na Tabela 2. A relação entre altura e diâmetro apresenta variância constante na maioria das vezes, portanto, o método utilizado no ajuste dos modelos aos dados foi o de máxima verossimilhança, por meio de modelo linear generalizado com resposta normal (ou gaussiana), para os modelos (1), (2), (3) e (4). Já, os modelos (5), (6) e (7) promovem a transformação da variável resposta altura para a escala logarítmica; para evitar essa mudança de escala, optou-se pelo ajuste desses modelos, utilizando o estimador de máxima verossimilhança com distribuição gama e função de ligação logarítmica (FARAWAY, 2006). Utilizando esse método, conseguiram-se estimativas dos parâmetros bem próximas às que se pode obter por meio da transformação da variável aleatória; porém, sem o inconveniente da mudança de escala. Para garantir a premissa de homocedasticidade, realizou-se o teste de Breusch-Pagan (BREUSCH; PAGAN, 1979) a 5\% de significância nos resíduos dos modelos de relação hipsométricas.

TABELA2 Modelos de relação hipsométrica e volumétrica testados em nove espécies arbóreas.

TABLE 2 Allometric models tested for nine tree species.

\begin{tabular}{lcc}
\hline Modelos hipsométricos & Autores & Modelo \\
\hline$h_{i}=\beta_{0}+\beta_{1} d_{i}+\varepsilon_{i}$ & Linha Reta \\
$h_{i}=\beta_{0}+\beta_{1} d_{i}+\beta_{2} d_{i}^{2}+\varepsilon_{i}$ & Parabólico \\
$h_{i}=\beta_{0}+\beta_{1} \frac{1}{d_{i}}+\beta_{2} \frac{1}{d_{i}^{2}}+\varepsilon_{i}$ & Henricksen \\
$h_{i}=\beta_{0}+\beta_{1} \ln \left(d_{i}\right)+\varepsilon_{i}$ & Curtis \\
$\ln \left(h_{i}\right)=\beta_{0}+\beta_{1} \frac{1}{d_{i}}+\varepsilon_{i}$ & Stoffels \\
$\ln \left(h_{i}\right)=\beta_{0}+\beta_{1} \ln \left(d_{i}\right)+\varepsilon_{i}$ & $(6)$ \\
$\ln \left(h_{i}\right)=\beta_{0}+\beta_{1} \ln \left(d_{i}\right)+\ln ^{2}\left(d_{i}\right)+\varepsilon_{i}$ & $(7)$ \\
\hline $\operatorname{Modelos} \operatorname{volumétricos}$ & Spurr \\
\hline$v_{i}=\beta_{0}+\beta_{1} d_{i}^{2} h_{i}+\varepsilon_{i}$ & Stoate \\
$v_{i}=\beta_{0}+\beta_{1} d_{i}^{2}+\beta_{2} d_{i}^{2} h_{i}+\beta_{3} h_{i}+\varepsilon_{i}$ & $(8)$ \\
$v_{i}=\beta_{0}+\beta_{1} d_{i}^{2}+\beta_{2} d_{i}^{2} h_{i}+\beta_{3} d_{i} h_{i}^{2}+\beta_{4} h_{i}^{2}+\varepsilon_{i}$ & $\begin{array}{c}\text { Meyer } \\
\text { modificada }\end{array}$ \\
$\ln \left(v_{i}\right)=\beta_{0}+\beta_{1} \ln \left(d_{i}^{2} h_{i}\right)+\varepsilon_{i}$ & Spurr \\
$\ln \left(v_{i}\right)=\beta_{0}+\beta_{1} \ln \left(d_{i}\right)+\beta_{2} \ln \left(h_{i}\right)+\varepsilon_{i}$ & logarítmica \\
\hline$h=$ & Schumacher- \\
Hall
\end{tabular}

$h_{i}=$ altura total $(\mathrm{em} \mathrm{m}) ; d=$ diâmetro medido a $1,30 \mathrm{~m}$ de altura do solo $(\mathrm{em} \mathrm{cm}) ; v_{i}=$ volume individual; $\beta_{0}, \beta_{1}, \beta_{2}, \beta_{3}$ e $\beta_{4}=$ parâmetros a serem estimados; $\varepsilon_{\mathrm{i}}=$ erro aleatório $(i=1 \ldots N)$.

$h_{i}=$ total height $(\mathrm{m}), d_{i}=$ diameter at breast height $(\mathrm{cm}) ; v_{i}=$ individual volume; $\beta_{0}, \beta_{1}, \beta_{2}, \beta_{3}$ and $\beta_{4}=$ parameters to be estimated; $\varepsilon_{\mathrm{i}}=$ random error $(i=1 \ldots N)$.

A relação entre volume e diâmetro possui comportamento característico de aumento da variabilidade, conforme se aumenta o valor do volume (heterocedasticidade). Ao se utilizar o método de mínimos quadrados ordinários na estimação dos parâmetros dos modelos, os estimadores são nãoviesados, mas não de variância mínima (MONTGOMERY et al., 2006). Para contornar esse problema, utilizou-se o método de mínimos quadrados ponderados pelo inverso da resposta média nos modelos (8), (9) e (10), ou seja, as observações de maiores volumes foram penalizadas na 
contribuição do cálculo das estimativas dos parâmetros. Os pesos utilizados pelo método de mínimos quadrados ponderados dependem de como a variância se comporta, de acordo com o aumento da variável resposta. No caso de equações de volume individuais de árvores, o uso do preditor linear ao quadrado é a ponderação mais usual (BATISTA et al., 2004; CLUTTER et al., 1983). Da mesma forma como realizado para as relações hipsométricas, utilizou-se o modelo linear generalizado com variável resposta gama e função de ligação logarítmica para os modelos (11) e (12), ao invés de transformar a variável resposta.

Os modelos foram ajustados aos dados de cada espécie separadamente e comparados pelo Critério de Informação de Akaike (equação 1), dado por Hobbs e Hilborn (2006), sendo AIC o critério de informação de Akaike, $L(\theta \mid Y) \circ$ valor da máxima verossimilhança, associado às estimativas dos parâmetros do modelo e $K$ o número de parâmetros do modelo.

$A I C=-2 \cdot \ln [L(\theta \mid Y)]+2 \cdot K$

O valor de AIC representa a quantidade de informação perdida em relação à realidade do fenômeno estudado (HOBBS; HILBORN, 2006). Portanto, foi selecionado o modelo com o menorvalor de AIC para cada espécie estudada. Quando dois modelos apresentaram valores de AIC próximos, o melhor modelo foi escolhido com base no princípio da parcimônia, ou seja, o modelo que melhor descreve o fenômeno estudado com o menor número de parâmetros possível.

Após a seleção do melhor modelo para cada espécie, realizou-se uma análise de resíduos para verificar se $\circ$ modelo estava livre de tendências ao estimar valores extremos e calculou-se o erro padrão da estimativa na unidade original e percentual, dado pelas equações 2 e 3 , sendo $s_{y x} \circ$ erro padrão da estimativa, $y_{i} \circ$ valor da variável resposta (volume ou altura, conforme o modelo) observada, $\hat{y}_{i}$ o valor estimado pelo modelo, $n$ o número de observações, $p$ o número de parâmetros do modelo selecionado e $\bar{y}$ a média dos valores da variável observada.

$s y x=\sqrt{\frac{\sum_{i=1}^{n}\left(y_{i}-\hat{y}_{i}\right)^{2}}{n-p}}$

$\operatorname{syx}(\%)=\frac{s y x}{\bar{y}} 100$

\section{RESULTADOS E DISCUSSÃO}

Os modelos propostos foram adequados ao estudo das relações hipsométricas, sendo que o modelo (6) se mostrou o mais flexível, ajustando para três espécies distintas, seguidos pelos modelos (1) e (4), que se ajustaram para duas espécies distintas cada (Tabela 3$)$. Modelos mais simples como (1) e (6) descrevem bem o conjunto de dados, pois as relações hipsométricas ainda não mostram uma estagnação definitiva do crescimento, indicando que a floresta ainda não se encontra em seu estado de pleno desenvolvimento (Figura 1). O modelo (7) também se mostrou bem adequado, apresentando valores de AIC bem reduzidos, no entanto, utilizando o princípio da parcimônia, outros modelos mais simples foram escolhidos.

TABELA 3 Valores do Critério de Informação de Akaike calculados para cada modelo em cada espécie, onde ID é a letra identificadora de espécie (Tabela 1). Em negrito, tem-se a indicação do modelo que melhor descreve o comportamento dos dados para cada espécie. O número dos modelos correspondem àqueles da Tabela 2.

TABLE 3 Akaike information criteria values calculated for each model and species, where ID is the identification of the species. The best model is shown in bold.

\begin{tabular}{|c|c|c|c|c|c|c|c|c|c|c|c|c|}
\hline \multirow{2}{*}{ ID } & \multicolumn{7}{|c|}{ Modelos Hipsométricos } & \multicolumn{5}{|c|}{ Modelos Volumétricos } \\
\hline & 1 & 2 & 3 & 4 & 5 & 6 & 7 & 8 & 9 & 10 & 11 & 12 \\
\hline$A$ & 510,8 & 511,1 & 515,1 & 521,3 & 540,4 & 514,2 & 513,3 & $-583,1$ & $-606,3$ & $-597,4$ & $-590,4$ & $-607,7$ \\
\hline B & 1187,7 & 1187,9 & 1191,8 & 1195,0 & 1183,2 & 1154,6 & 1153,0 & $-1369,4$ & $-1518,4$ & $-1491,3$ & $-1417,6$ & $-1534,8$ \\
\hline $\mathrm{C}$ & 528,7 & 528,7 & 528,7 & 527,9 & 532,0 & 524,5 & 526,5 & $-635,0$ & $-665,4$ & $-653,9$ & $-639,6$ & $-665,7$ \\
\hline $\mathrm{D}$ & 591,3 & 591,8 & 591,5 & 594,5 & 599,2 & 584,1 & 584,8 & $-954,7$ & $-982,5$ & $-965,6$ & $-963,6$ & $-980,7$ \\
\hline$E$ & 459,4 & 453,8 & 455,3 & 451,9 & 453,0 & 460,5 & 454,1 & $-347,8$ & $-404,6$ & $-403,0$ & $-359,9$ & $-401,7$ \\
\hline $\mathrm{F}$ & 100,3 & 102,1 & 101,3 & 103,9 & 107,2 & 103,1 & 102,7 & $-215,5$ & $-223,1$ & $-220,0$ & $-217,0$ & $-225,2$ \\
\hline G & 269,2 & 266,8 & 266,9 & 266,6 & 270,0 & 268,0 & 269,9 & $-576,1$ & $-589,8$ & $-597,7$ & $-577,3$ & $-606,1$ \\
\hline $\mathrm{H}$ & 113,5 & 115,0 & 114,3 & 113,0 & 112,8 & 113,2 & 114,6 & $-244,0$ & $-244,3$ & $-240,2$ & $-240,9$ & $-245,1$ \\
\hline I & 621,8 & 633,8 & 639,2 & 634,3 & 612,7 & 612,5 & 614,4 & $-1406,0$ & $-1442,1$ & $-1424,3$ & $-1402,0$ & $-1453,2$ \\
\hline
\end{tabular}



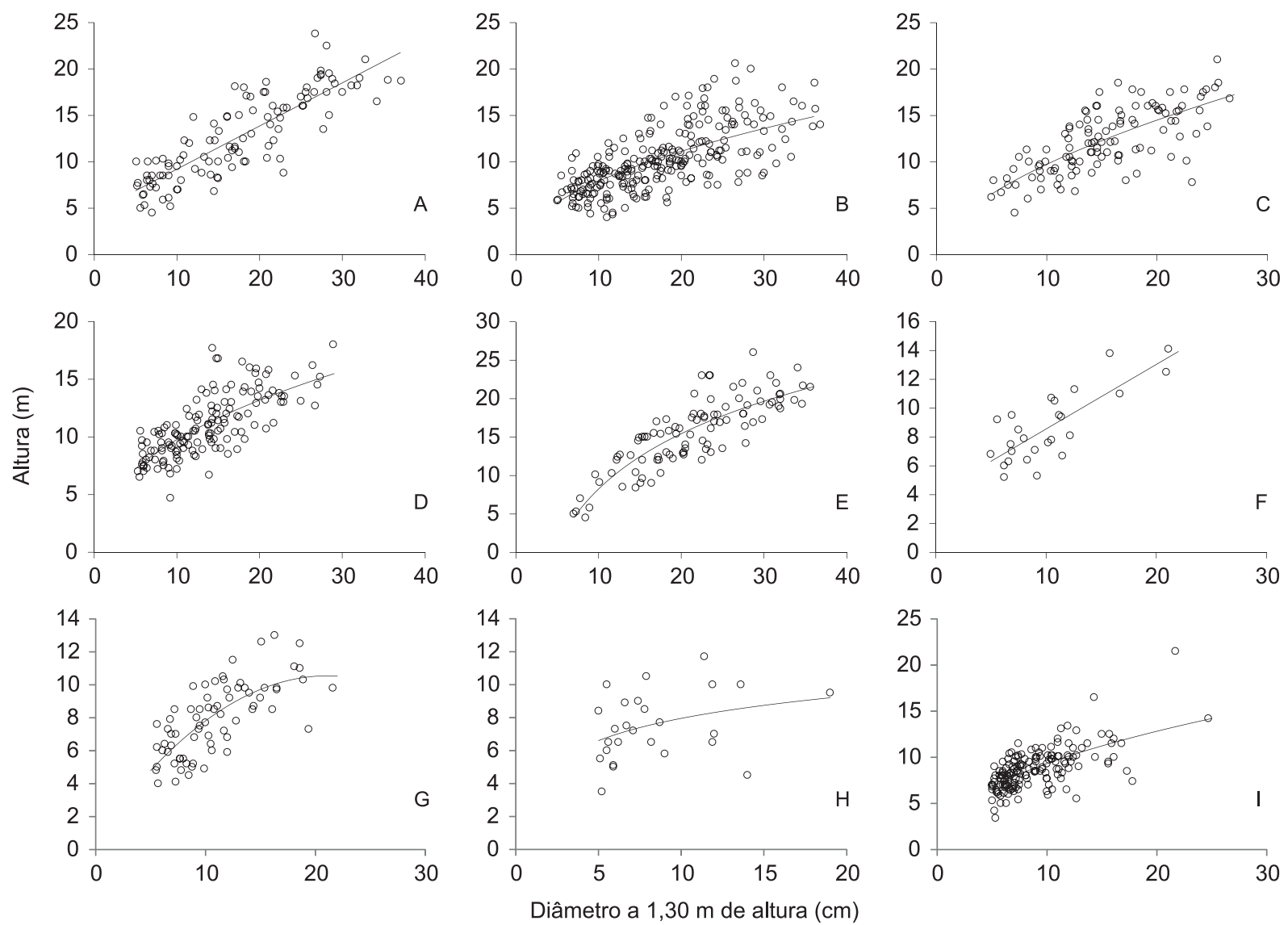

FIGURA 1 Relações entre altura e diâmetro para todas as árvores das nove espécies no presente estudo. A letra em cada gráfico identifica a espécie (Tabela 1).

FIGURE 1 Height-diameter relationship for all trees of nine species of the present study. The letter in each graph identifies the species (Table 1).

$\mathrm{Na}$ Tabela 4, os resultados dos testes de Breusch-Pagan, indicaram homocedasticidade dos modelos a $5 \%$ de significância. A precisão média das equações, representada pelo erro padrão da estimativa $\left(s_{y x}\right)$, situou-se entre I,5I e 2,5I metros. Esses valores são um pouco elevados se comparados aos estudos com florestas homogêneas (BARROS et al., 2002; BATISTA et al., 2004; FINGER et al., 2000; ZANON et al., 1996), que costumam se situar entre I, 0 e I, $5 \mathrm{~m}$. Em florestas mistas, há uma maior heterogeneidade no desenvolvimento dos indivíduos, causada pela competição interespecífica e pela própria variabilidade genética existente entre indivíduos de mesma espécie, contribuindo para o aumento do valor do erro padrão da estimativa (COLPINI et al., 2009; ZANON et al., 1996). Já, os valores percentuais, além de acompanhar os elevados valores de erros padrão residuais absolutos, contribuem com um baixo valor da média das alturas, pois a distribuição das alturas é assimétrica, também típica de florestas mistas.

$O$ erro padrão residual em percentagem para as espécies $E$. contorstisiliquum e $C$. langsdorffii foi bem elevado, em razão de seu padrão de ramificação, descaracterizando sua relação entre diâmetro e altura (Tabela 4). Observações em campo mostram que o padrão de crescimento em altura dos indivíduos que se desenvolvem nas bordas do experimento e, consequentemente, a forma da árvore, é totalmente diferente daqueles que desenvolvem no interior, com menor incidência lateral de luz. Essas alterações na arquitetura da árvore contribuem, substancialmente, com o aumento do erro padrão residual.

$\mathrm{Na}$ análise de resíduos dos modelos hipsométricos (Figura 2), pode-se constatar que os modelos se ajustaram bem aos dados, não havendo problemas de tendência em valores extremos e autocorrelação. Foi detectada a presença de pontos discrepantes, ou seja, com resíduos superiores a $2 \mathrm{em}$ módulo. Esse comportamento já era esperado, em razão da alta variabilidade das relações entre diâmetro e altura em florestas nativas. Optou-se por não remover os pontos da análise por se caracterizar um fenômeno natural, nesse caso. 
TABELA 4 Estimativas dos parâmetros, erros padrões de estimativa, $R^{2}$ ajustado para os modelos lineares e o $p$-valor para o teste de homocedasticidade (BP) para os modelos hipsométricos selecionados para cada espécie.

TABLE 4 Parameters estimates, residual standard errors, adjusted $\mathrm{R}^{2}$ and homoscedasticity test (BP) for the hypsometric models for each species.

\begin{tabular}{lcccccccc}
\hline Espécie & Modelo & $\hat{\beta}_{0}$ & $\hat{\beta}_{1}$ & $\hat{\beta}_{2}$ & $s_{y x}$ & $s_{y x} \%$ & $\mathrm{R}^{2}{ }_{\text {aj }}$ & $\mathrm{BP}$ \\
\hline A. macrocarpa & $(1)$ & 4,566 & 0,465 & - & 2,43 & 19,16 & 0,72 & 0,110 \\
E. contorstisiliquum & $(6)$ & 0,937 & 0,492 & - & 2,44 & 24,07 & - & 0,807 \\
P. dubium & $(6)$ & 0,951 & 0,575 & - & 2,41 & 19,63 & - & 0,949 \\
P. nitens & $(6)$ & 1,283 & 0,433 & - & 1,81 & 16,51 & - & 0,639 \\
S. parahyba & $(4)$ & $-15,636$ & 10,376 & - & 2,51 & 16,05 & 0,73 & 0,659 \\
Z. tuberculosa & $(1)$ & 4,071 & 0,448 & - & 1,54 & 17,78 & 0,61 & 0,846 \\
C. fissilis & $(2)$ & 0,673 & 0,936 & $-0,022$ & 1,53 & 19,32 & 0,51 & 0,591 \\
C. langsdorffii & $(4)$ & 3,475 & 1,945 & - & 1,97 & 26,31 & 0,08 & 0,489 \\
H. courbaril & $(6)$ & 1,176 & 0,459 & - & 1,71 & 19,55 & - & 0,208 \\
\hline
\end{tabular}

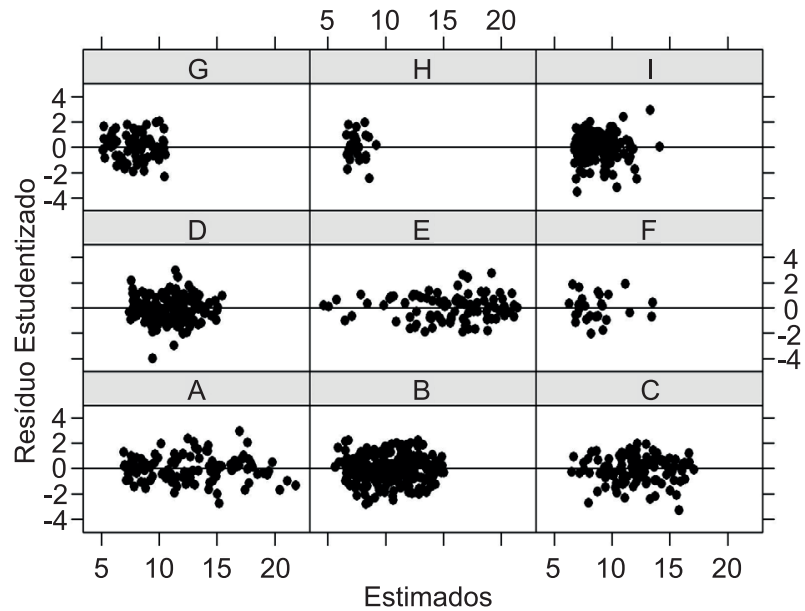

FIGURA 2 Análise de resíduos dos modelos hipsométricos ajustados, onde as letras de $\mathrm{A}$ a I representam as nove espécies estudadas.

FIGURE 2 Residual analysis of the fitted hypsometric models, where the letters A to I are the nine species studied.
Quanto aos modelos volumétricos, o modelo (12) foi o que se mostrou mais flexível, ajustando-se melhor em sete das nove espécies estudadas (Tabela 5). Esse modelo, também conhecido como modelo de Schumacher-Hall, tem sido empregado amplamente para estimação de volume de árvores, em razão de sua versatilidade, tanto para espécies crescendo em monocultivo como em plantios mistos e florestas nativas (AKINDELE; LEMAY, 2006; CHICHORRO et al., 2003; COLPINI et al., 2009; SILVA; CARVALHO, 1984; SILVA et al., 1984).

De maneira geral, os valores de $s_{y x}$ (Tabela 5) são considerados bem baixos, conforme comparação com a literatura consultada (IMAÑA-ENCINAS et al., 2009; SCOLFORO et al., 1994; SILVA; CARVALHO, 1984; SILVA et al., 1984; TONINI et al., 2005; TONINI; SCHWENGBER, 2006). No entanto, os valores percentuais aparentam serem elevados, em decorrência da presença de muitas árvores de pequenas dimensões

TABELA 5 Estimativas dos parâmetros e erros padrões de estimativa para os modelos volumétricos selecionados para cada espécie.

TABLE 5 Parameters estimates and residual standard errors for the volumetric models for each species.

\begin{tabular}{|c|c|c|c|c|c|c|c|}
\hline Espécie & Modelo & $\hat{\beta}_{0}$ & $\hat{\beta}_{1}$ & $\hat{\beta}_{2}$ & $\hat{\beta}_{3}$ & $s_{y x}$ & $s_{y x} \%$ \\
\hline Anadenanthera macrocarpa & $(12)$ & $-9,7471$ & 2,1260 & 0,6300 & - & 0,0418 & 22,68 \\
\hline Enterolobium contorstisiliquum & $(12)$ & $-9,4143$ & 2,1203 & 0,4774 & - & 0,0322 & 23,46 \\
\hline Peltophorum dubium & $(12)$ & $-9,6137$ & 2,1604 & 0,5373 & - & 0,0266 & 23,18 \\
\hline Pterogyne nitens & (9) & $-2,5910^{-3}$ & $1,5610^{-4}$ & $1,8010^{-5}$ & $2,2310^{-4}$ & 0,0176 & 21,50 \\
\hline Schizolobium parahyba & (9) & $-4,0210^{-3}$ & $3,3310^{-4}$ & $1,6910^{-5}$ & $-1,2410^{-3}$ & 0,0521 & 17,91 \\
\hline Zeyheria tuberculosa & $(12)$ & $-9,6752$ & 2,1323 & 0,5919 & - & 0,0095 & 22,17 \\
\hline Cedrela fissilis & $(12)$ & $-9,7095$ & 2,1677 & 0,5761 & - & 0,0061 & 13,78 \\
\hline Copaifera langsdorffii & $(12)$ & $-9,4651$ & 1,9208 & 0,6900 & - & 0,0051 & 22,99 \\
\hline Hymenaea courbaril & (12) & $-9,7326$ & 2,1729 & 0,5887 & - & 0,0099 & 31,32 \\
\hline
\end{tabular}


(Figura 1-B), contribuindo com o valor reduzido da média do volume individual no cômputo do erro padrão residual em percentagem $\left(s_{y x} \%\right)$.

Os gráficos de resíduos dos modelos volumétricos (Figura 3) indicam boa distribuição de resíduos, indicando ajuste adequado para a maioria das espécies. Observou-se a presença de alguns pontos discrepantes, mas que não alteraram significativamente a estrutura da relação, por isso foram mantidos.

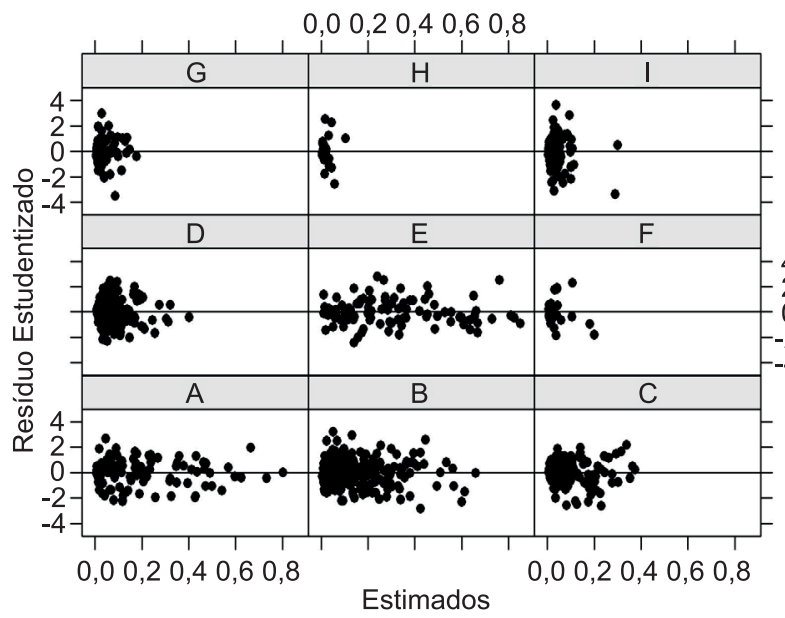

FIGURA 3 Análise de resíduos dos modelos volumétricos ajustados, onde as letras de $\mathrm{A}$ a I representam as nove espécies estudadas.

FIGURE 3 Residual analysis of the fitted volumetric models, where the letters A to I are the nine species studied.

\section{CONCLUSÃO}

Neste trabalho, foi possível modelar as relações hipsométricas e volumétricas de nove espécies da floresta estacional semidecidual implantadas em diferentes sistemas alternativos de restauração florestal. Os modelos apresentados atenderam às expectativas e foi possível encontrar pelo menos um modelo, para cada espécie, que se ajustou satisfatoriamente e descreveu as relações alométricas estudadas.

Essas equações, que podem ser livremente empregadas nos modelos alternativos de restauração estudados, serão muito úteis em estudos futuros de manejo e conservação dessas áreas.

\section{AGRADECIMENTOS}

À Fapesp e CNPq pelo apoio financeiro e bolsa de produtividade em pesquisa para a segunda autora. Os autores agradecem integrantes do Laboratório de Ecologia e Restauração Florestal (LERF/UNESP) pela ajuda em campo, principalmente a Elder C. Mattos, e aos professores Dr. Cláudio R. Thiersch, Dra. Liciana V. de A. Silveira e Dr. João L. F. Batista pelas sugestões de análise estatística.

\section{REFERÊNCIAS}

AKINDELE, S. O.; LEMAY, V. M. Development of tree volume equations for commom timber species in tropical rain forest area of Nigeria. Forest Ecology and Management, Amsterdam, v. 226, p. 4I-48, 2006.

BARROS, D. A.; MACHADO, A. S.; ACERBI JÚNIOR, F. W.; SCOLFORO, J. R. Comportamento de modelos hipsométricos tradicionais e genéricos para plantações de Pinus oocarpa em diferentes tratamentos. Boletim de Pesquisa Florestal, Colombo, v. 45, p. 3-28, 2002.

BATISTA, J. L. F;; MARCHESINI, M.; VIANA, V. M. Equações de volume para árvores de caxeta (Tabebuia cassinoides) no Estado de São Paulo e sul do Estado do Rio de Janeiro. Scientia Forestalis, Piracicaba, v. 65, p. I62-175, 2004.

BREUSCH, T. S.; PAGAN, A. R. A simple test for heteroscedasticity and random coefficient variation. Econometrica, Chichester, v. 47, p. I287-1294, 1979.

CHICHORRO, J. F.; RESENDE, P. J. L.; LEITE, G. A. Equações de volume e de taper para quantificar multiprodutos da madeira em floresta atlântica. Revista Árvore, Viçosa, v. 27, n. 6, p. 799-809, nov./dez. 2003.

CLUTTER, J.; FORTSON, J. C.; PIENAAR, L. V.; BRISTER, G. H.; BAILEY, R. L. Timber management: a quantitative approach. New York: J. Wiley, 1983.

COLPINI, C.; TRAVAGINI, P. D.; SOARES, S. T. Determinação do volume, do fator de forma e da porcentagem de casca de árvores individuais em uma floresta ombrófila aberta na região noroeste de Mato Grosso. Acta Amazônica, Manaus, v. 39, n. I, p. 97-104, 2009.

CURTIS, R. O. Height-diameter and height-diameter-age equations for second-growth douglas-fir. Forest Science, Bethesda, v. I3, n. 4, p. 365-375, 1967.

DEAN, W. A ferro e fogo: a história e a devastação da mata atlântica brasileira. São Paulo: Companhia das Letras, 2007. $484 \mathrm{p}$.

ENGEL, V. L. Manejo sustentável e restauração florestal. In: SEMINÁRIO TEMÁTICO SOBRE RECUPERAÇÃO DE ÁREAS DEGRADADAS, 2003, São Paulo. Anais... São Paulo: Instituto de Botânica, 2003. p. 2I-3I.

FARAWAY, J. Extending the linear model with R: generalized linear, mixed effects and nonparametric regression models. London: Chapman \& Hall/CRC, 2006. 33I p. 
FINGER, C. A. G.; SPATHELF, P.; SCHNNEIDER, P. R.; COELHO, L. Curvas de altura-diâmetro de acácia negra (Acacia mearnsii de Wild). Ciência Rural, Santa Maria, v. 30, n. 3, p. 387-39I, maio/jun. 2000.

HOBBS, N. T.; HILBORN, R. Alternatives to statistical hypothesis testing in ecology: a guide to self teaching. Ecological Applications, Washington, v. 16, n. I, p. 5-19, 2006.

IMAÑA-ENCINAS, J.; SANTANA, O. A.; PAULA, J. E.; IMAÑA, C. R. Equações de volume de madeira para o cerrado de Planaltina de Goiás. Floresta, Curitiba, v. 39, n. I, p. I07II6, 2009.

INSTITUTO BRASILEIRO DE GEOGRAFIA E ESTATÍSTICA. Mapa de vegetação do Brasil. Rio de Janeiro, 1993. I:5.000.000.

LAMB, D.; ERSKINE, P. D.; PARROTA, J. A. Restoration of degraded tropical forest landscapes. Science, New York, v. 310, p. 1628-1632, 2005.

MELO, A. C. G.; DURIGAN, G. Evolução estrutural de reflorestamentos de restauração de matas ciliares no médio do vale do Paranapanema. Scientia Forestalis, Piracicaba, v. 73, p. I0I-III, 2007.

MONTGOMERY, D. C.; PECK, E. A.; VINNING, G. G. Introduction to linear regression analysis. $4^{\text {th }}$ ed. New York: Wiley-Interscience, 2006. 612 p.

RÉ, D. S. Equações alométricas em plantios mistos visando à restauração da floresta estacional semidecidual. 2011. 57 p. Dissertação (Mestrado em Ciência Florestal) - Universidade Estadual Paulista "Júlio de Mesquita Filho", Botucatu, 20I I.
SCOLFORO, J. R. S. Biometria florestal: parte I: modelos de regressão linear e não linear: parte II: modelos de relação hipsométrica, volume, afilamento e peso de matéria seca. Lavras: UFLA/FAEPE, 2005. 352 p.

SCOLFORO, J. R. S.; MELLO, J. M.; LIMA, C. S. A. Obtenção de relações quantitativas para a estimativa de volume do fuste em floresta estacional semidecídua montana. Cerne, Lavras, v. I, n. I, p. 123-134, 1994.

SILVA, J. N. M.; CARVALHO, M. S. P. Equações de volume para uma floresta secundária no planalto do Tapajós-Belterra, PA. Boletim de Pesquisa Florestal, Colombo, v. 8/9, p. I-I5, 1984.

SILVA, J. N. M.; CARVALHO, J. O. P.; LOPES, J. C. A.; CARVALHO, M. S. P. Equações de volume para a floresta nacional do Tapajós. Boletim de Pesquisa Florestal, Colombo, v. 8/9, p. 50-63, 1984.

TONINI, H.; ARCO VERDE, M. F.; SÁ, S. P. P. Dendrometria de espécies nativas em plantios homogêneos no Estado de Roraima - Andiroba (Carapa guianensis Aubl), Castanhado-Brasil (Bertholletia excelsa Bonpl.), Ipê-roxo (Tabebuia avellanedae Lorentz ex Griseb) e Jatobá (Hymenaea courbaril L.). Acta Amazônica, Manaus, v. 35, n. 3, p. 353362, 2005.

TONINI, H.; SCHWENGBER, L. A. M. Equações hipsométricas e volumétricas para Acacia mangium Willd em Roraima. Ambiência, Varela de Sá, v. 2, n. 2, p. I55-165, 2006.

ZANON, M. L. B.; FINGER, C. A. G.; SCHNEIDER, P. R.; KLEIN, J.; MEYER, E.; COELHO, M. C. B. Funções para descrever a relação altura diâmetro de Eucalyptus dunnii Maiden. Ciência Rural, Santa Maria, v. 26, n. I, p. 87-90, jan./fev. 1996. 